\title{
Induced Pluripotent Stem Cells from Human Placental Chorion for Perinatal Tissue Engineering Applications
}

\author{
Guihua Jiang, MS, ${ }^{1,2}$ Julie Di Bernardo, $\mathrm{PhD},{ }^{2}$ Cynthia J. DeLong, $\mathrm{PhD},{ }^{1,3}$ \\ André Monteiro da Rocha, $\mathrm{PhD},{ }^{1,4}$ K. Sue O'Shea, $\mathrm{PhD}{ }^{1,3}$ and Shaun M. Kunisaki, MD, MSc $c^{1,2,4}$
}

The reliable derivation of induced pluripotent stem cells (iPSCs) from a noninvasive autologous source at birth would facilitate the study of patient-specific in vitro modeling of congenital diseases and would enhance ongoing efforts aimed at developing novel cell-based treatments for a wide array of fetal and pediatric disorders. Accordingly, we have successfully generated iPSCs from human fetal chorionic somatic cells extracted from term pregnancies by ectopic expression of OCT4, SOX2, KLF4, and cMYC. The isolated parental somatic cells exhibited an immunophenotypic profile consistent with that of chorionic mesenchymal stromal cells (CMSCs). CMSC-iPSCs maintained pluripotency in feeder-free systems for more than 15 passages based on morphology, immunocytochemistry, and gene expression studies and were capable of embryoid body formation with spontaneous trilineage differentiation. CMSC-iPSCs could be selectively differentiated in vitro into various germ layer derivatives, including neural stem cells, beating cardiomyocytes, and definitive endoderm. This study demonstrates the feasibility of term placental chorion as a novel noninvasive alternative to dermal fibroblasts and cord blood for human perinatal iPSC derivation and may provide additional insights regarding the reprogramming capabilities of extra-embryonic tissues as they relate to developmental ontogeny and perinatal tissue engineering applications.

\section{Introduction}

$\mathbf{T}$ HE SEMINAL WORK by Yamanaka and colleagues, among others, on reprogramming somatic cells into a pluripotent state has reinvigorated the field of stem cell biology. ${ }^{1,2}$ Pluripotency and the indefinite capacity for selfrenewal similar to embryonic stem cells (ESCs) can be achieved with mouse embryonic fibroblasts (MEFs) as well as human dermal fibroblasts by forced expression of four transcription factors: OCT4, SOX2, KLF4, and cMYC (OSKM). Patient-specific induced pluripotent stem cell (iPSC) technologies can provide a useful model to study disease pathogenesis of specific tissues, particularly for disorders in which no suitable in vitro or animal model currently exists. ${ }^{3}$ Moreover, the generation of iPSCs has provided new opportunities for the development of fetal cell-based therapies, an area within regenerative medicine that has been hampered by a general lack of reliable and ethically acceptable alternative to human ESCs for transplantation purposes.

Since these initial reports, other somatic cells obtained from tissues, including liver, fat, and blood, have been re- programmed into iPSCs using defined factors. ${ }^{4,5}$ The placenta, an extra-embryonic organ that is traditionally discarded at birth, has only recently been explored for its pluripotency and iPSC reprogramming capabilities. ${ }^{6-8}$ Since the chorionic layer represents the predominant mass fraction of the placental membrane, ${ }^{6,9}$ we sought to generate new iPSC lines derived from term human placental chorionic mesenchymal stromal cells (CMSCs) and to explore their therapeutic potential by controlled and selective differentiation into various germ layer derivatives in vitro.

\section{Materials and Methods}

\section{Isolation of placental CMSCs}

The ethical approval was given by the Institutional Review Board and the Human Pluripotent Stem Cell Research Oversight Committee at the University of Michigan (No. 38565). After written informed consent, human placentae $(n=9)$ were retrieved fresh from healthy uncomplicated pregnancies following elective cesarean section at the Von Voigtlander Women's Hospital, University of Michigan Health System. The chorion was mechanically separated

\footnotetext{
${ }^{1}$ From the Consortium for Stem Cell Therapies, C.S. Mott Children's Hospital, Von Voigtlander Women's Hospital, University of Michigan Medical School, Ann Arbor, Michigan.

Departments of ${ }^{2}$ Surgery, ${ }^{3}$ Cell and Developmental Biology, and ${ }^{4}$ Obstetrics and Gynecology, C.S. Mott Children's Hospital, Von Voigtlander Women's Hospital, University of Michigan Medical School, Ann Arbor, Michigan.
} 
from the amnion and chorionic villi and decidua. Specimens were washed and enzymatically digested in collagenase $(1.25 \mathrm{mg} / \mathrm{mL}$; Worthington Biochemical) and dispase (1.2 U/mL; Worthington Biochemical) for $1-2 \mathrm{~h}$ at $37^{\circ} \mathrm{C}$ with agitation. Cells were washed twice before resuspension in mesenchymal basal medium (DMEM, 10\% fetal bovine serum, $100 \mathrm{U} / \mathrm{mL}$ penicillin and $100 \mu \mathrm{g} / \mathrm{mL}$ streptomycin, and $2 \mathrm{mM}$ L-glutamine; Sigma-Aldrich).

\section{Characterization of CMSCs}

CMSCs between passages 3 and 5 were evaluated for MSC characteristics based on criteria as described elsewhere. ${ }^{10}$ For flow cytometry, the primary antibodies used were phycoerythron-conjugated against CD34, CD44, CD45, CD73, CD90, CD105, OCT4A, SOX2, SSEA3, SSEA4, TRA-1-60, TRA-181, and HLA-DR (all from BD Biosciences). Evaluation of antibody staining was performed using the LSRII flow cytometer (BD Biosciences), and the resultant data were analyzed using FloJo software (Tree Star). To evaluate the proliferation and clonogenic capacity of CMSCs in culture, colony-forming unit-fibroblast (CFU-F) assays were performed in triplicate as previously described. ${ }^{11}$ Adipocyte and osteoblast differentiation of CMSCs was performed using established protocols. ${ }^{12}$

\section{iPSC derivation and analysis}

iPSC derivation was performed by ectopic Yamanaka factor reprogramming techniques as described elsewhere. ${ }^{11,13}$ Briefly, 293FT cells (Invitrogen) were transfected by the calcium phosphate precipitation method with pUMVC and pCMVVSV-g plasmids, in addition to pMX-hKLF4, -hSOX2, -hOCT4, -hcMYC, and -RFP plasmids, at a 1:1:1 ratio. The supernatant was used to transduce three randomly selected CMSC samples (CMSC-1, CMSC-2, and CMSC-3) at passage 4 at a multiplicity of infection of 5 . At day 6 , the cells were split and placed on irradiated MEFs feeder layers. All cells, including H9-ESCs controls, were cultured in human ES conditioned medium (GlobalStem) with $4 \mathrm{ng} / \mathrm{mL}$ of FGF-2 (Millipore). Individual colonies derived from CMSCs were subsequently picked and propagated on MEFs, Matrigel (BD Biosciences), or PMEDSAH, a novel and defined synthetic matrix polymer. ${ }^{11}$ Alkaline phosphatase staining was performed using the AP substrate kit (Vector Laboratories) according to the manufacturer's instructions. Embryoid bodies (EBs) were formed in suspension culture and analyzed based on the methods described previously. ${ }^{14}$

Total RNA was reverse transcribed into cDNA before amplification with the appropriate positive and negative controls in triplicate to 35 cycles (Life Technologies). The primer sequences for reverse transcription polymerase chain reaction (RT-PCR) are shown in Supplementary Table S1 (Supplementary Data are available online at www.liebertpub.com/tec). Relative gene expression was analyzed by quantitative polymerase chain reaction (qPCR), with GAPDH used as a reference gene for the normalization of target gene expression using the $2^{-\Delta \Delta \mathrm{Ct}}$ method. ${ }^{15}$ The primer sequences used for $\mathrm{qPCR}$ are shown in Supplementary Table S2.

To assay pluripotency, primary antibodies against NANOG (Abcam), OCT4 (Santa Cruz), SOX2, SSEA3, SSEA4, TRA-1-60, and TRA-1-81 (Millipore) were used. Primary antibodies used to evaluate the differentiation were against Nestin (Millipore), Neuron-specific class beta III tubulin (TuJ1; Covance), PSA-NCAM (Millipore), $\alpha$-actinin (Sigma-Aldrich), Myosin heavy chain (MF20 and MYH7), MLC2 $v$ (Proteintech), $\alpha$-smooth muscle actin ( $\alpha$-SMA), alpha fetoprotein (AFP), FOXA2, and SOX17 (R\&D Systems). Cells were examined by epifluorescence microscopy (Leica). Karyotyping was performed on CMSC-iPSC lines every five passages by Cell Line Genetics, Inc.

\section{Neural differentiation}

Neural differentiation of iPSCs was conducted based on modifications of a protocol described elsewhere. ${ }^{16}$ Briefly, CMSC-iPSC colonies at passage 7 were manually cut and transferred into 60-mm Petri dishes for 4 days followed by culture in the neuron induction medium (DMEM/F12, GlutaMAX, NEAA, and N2; Invitrogen) for 2 days. The resulting neurospheres were transferred into polyornithine/ laminin-coated six-well plates (Sigma-Aldrich) to facilitate rosette formation. Individual rosettes were manually isolated, dissected, and replated into dishes containing neural stem cell (NSC) media (Neurobasal media, B27 [Invitrogen], NEAA, GlutaMAX, and FGF-2).

\section{Cardiac differentiation}

Cardiomyocyte differentiation of iPSCs was performed using the Matrigel sandwich technique as previously detailed. ${ }^{17}$ Briefly, CMSC-iPSC clones at passage 14 were incubated with TrypleE (Invitrogen) at $37^{\circ} \mathrm{C}$ for $5 \mathrm{~min}$ to obtain a single-cell suspension. The cells were seeded into Matrigel-coated 12well plates at a density of 100,000 cells $/ \mathrm{cm}^{2}$ in mTESR1 medium (StemCell Technologies) and grown to near confluence, at which time the wells were overlaid with additional Matrigel. Once the cells were $100 \%$ confluent, the media was replaced with RPMI 1640 (Invitrogen) containing B27 and $100 \mathrm{ng} / \mathrm{mL}$ activin A (R\&D Systems). After $24 \mathrm{~h}$, the media was supplemented with $10 \mathrm{ng} / \mathrm{mL}$ BMP4 (R\&D Systems) and FGF-2 for another 4 days.

\section{Definitive endodermal differentiation}

Directed differentiation of iPSCs toward a definitive endodermal (DE) phenotype was performed based on published methods. ${ }^{18,19}$ Briefly, CMSC-iPSC colonies between passages 11 and 14 were exposed to dispase, triturated, and plated in Matrigel-coated 24-well plates. When 85-90\% confluent, the cells were cultured in endodermal differentiation media containing RPMI 1640, $100 \mathrm{ng} / \mathrm{mL}$ activin A, and $0-2 \%$ fetal bovine serum for 3 days and analyzed by immunofluorescence staining and gene expression as described previously.

\section{Statistical analyses}

Quantitative data were performed in triplicate and presented as the mean \pm SEM. The data were analyzed by one-way analysis of variance with Bonferroni correction for multiple comparisons using GraphPad Prism software (version 5.0d). Results were considered to be statistically significant if $p \leq 0.05$.

\section{Results}

Plastic adherent fibroblast-appearing cells were detected from all specimens (mean gestational age, 37 weeks) within 
1 week after seeding. The cells grew in monolayers and displayed a relatively homogeneous spindle-shaped morphology after the first passage (Fig. 1A, upper left). In seven of nine (78\%) samples, cell lines were successfully cultured for up to six passages at a ratio of 1:4 without evidence of reduced cell proliferation. Lack of maternal contamination among CMSCs from male neonates was confirmed by SRY and X-linked amelogenin gene expression (Supplementary Fig. S1).

Chorionic cells were clonogenic, demonstrating a CFU-F of $5.70 \pm 1.43$ colonies for every 100 cells plated (Fig. 1A, lower left). The mean number of placental chorion-derived cells obtained from a $1 \mathrm{~cm}^{2}$ biopsy was $1.1 \times 10^{8}$ cells (range, $8.0 \times 10^{6}-6.4 \times 10^{8}$ cells) after $26.1 \pm 3.1$ days in culture.
CMSCs were able to differentiate into various mesodermal cells types, namely adipocytes and osteocytes, when cultured in the appropriate directive media for 3 weeks (Fig. 1A, B). Evidence of adipogenic differentiation was indicated by the presence of Oil Red O-positive lipid vacuoles within the cytoplasm of a subpopulation of CMSCs (Fig. 1A, upper right) and by increased expression of adipocytespecific genes, including fatty acid binding protein-4 (FABP4, Fig. 1B, upper) and peroxisome proliferatoractivated receptor gamma (PPAR $\gamma$, data not shown), compared to CMSCs in the control medium. Osteogenic differentiation was less robust but was positive as shown by increased extracellular calcium matrix staining in the presence of Alizarin Red (Fig. 1A, lower right) and by

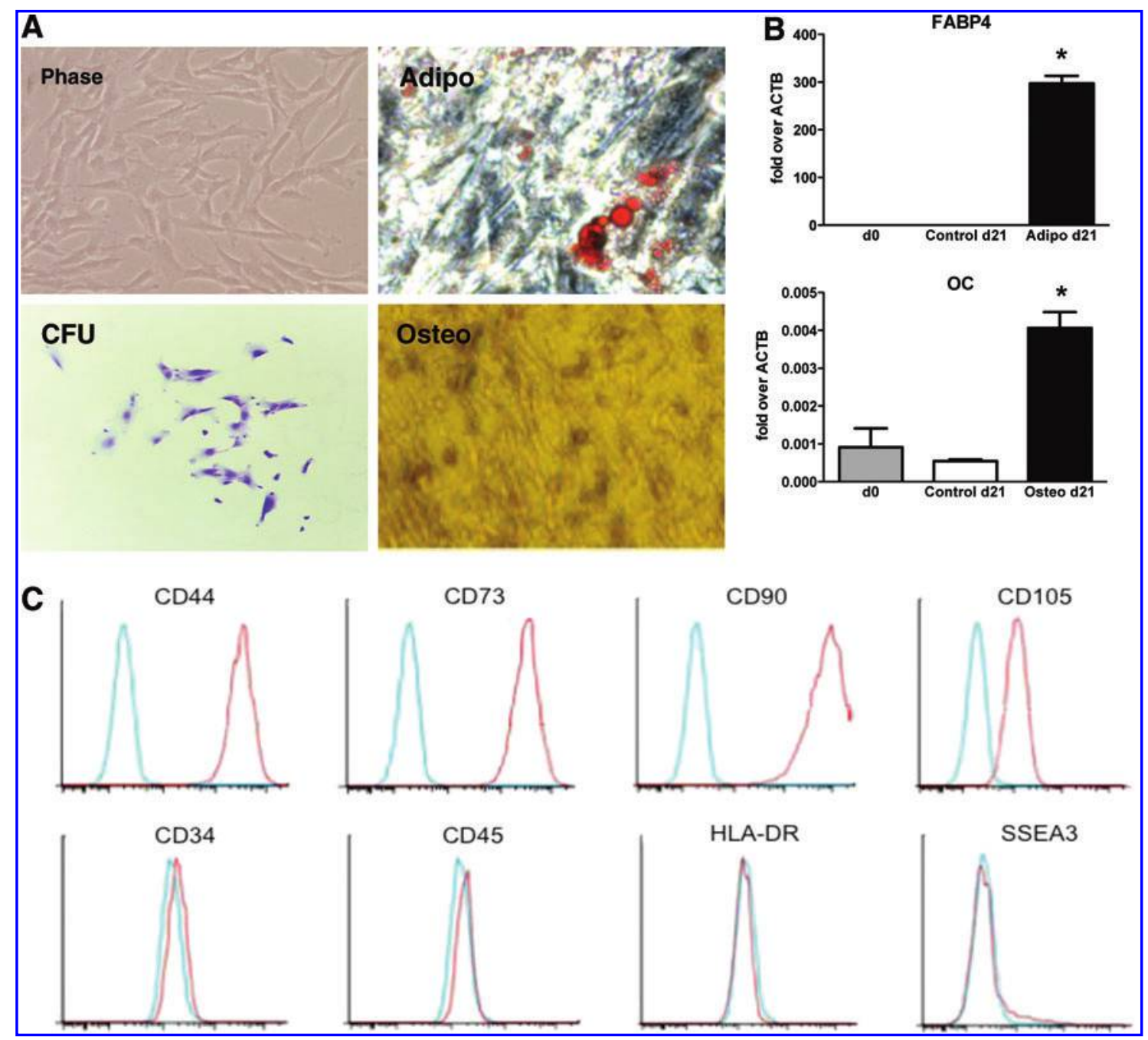

FIG. 1. Characterization of human chorionic mesenchymal stromal cells (CMSCs). (A) Phase-contrast photomicrograph illustrating typical CMSC morphology at passage 4 (upper left, magnification, $100 \times$ ). Representative colony-forming unitfibroblast (CFU-F) from CMSCs in serial dilution assays (lower left, magnification, $60 \times$ ). Oil Red O staining of CMSC-2 under adipogenic conditions for 21 days reveals orange-red cytoplasmic lipid droplets consistent with adipocytes (upper right, magnification, $100 \times$ ). Alizarin Red S staining of CMSC-2 in osteogenic conditions for 21 days shows early extracellular mineral deposition (lower right, magnification, $100 \times$ ). (B) Representative quantitative polymerase chain reaction (qPCR) demonstrates the upregulation of the adipogenic marker, fatty acid binding protein 4, (FABP4, upper), and the osteogenic marker, osteocalcin (OC, lower) in CMSC-2 under adipogenic and osteogenic conditions, respectively (*denotes $p \leq 0.05$ ). (C) Immunophenotypic characterization of CMSC-1 (shown in red compared with blue negative control) at passage 4 in mesenchymal basal media, confirming a mesenchymal stromal cell profile with positive staining for CD44, CD73, CD90, and CD105 (upper), and negative staining for CD34, CD45, HLR-DR, and SSEA3 (lower). Color images available online at www.liebertpub.com/tec 
expression of the late osteogenic-specific gene, osteocalcin (OC), compared to CMSCs in the control medium (Fig. 1B, lower).

Flow cytometry revealed that the immunophenotypic profile of culture-expanded CMSCs was consistent with that of MSCs derived from bone marrow and other postnatal sources (Fig. 1C). CMSCs highly expressed several mesenchymal surface epitope markers, including CD44 (99.2\% of cells), CD73 (98.7\%), CD90 (99.0\%), and CD105 (93.2\%). In contrast, less than $1 \%$ of cells expressed HLADR and hematopoietic stem cell markers, including CD34, CD45, and CD117. One percent of CMSCs expressed OCT4A, whereas $6.5 \%$ and $34.4 \%$ of cells displayed SSEA3 and SSEA4, respectively (data not shown).

The transfection efficiency of the reprogramming vectors based on RFP expression was $61.3 \% \pm 25.3 \%$ (Fig. 2A). After an initial period characterized by increased cell death, surviving CMSCs began to aggregate while reducing in size and losing their spindle-shaped morphology between 14 and 21 days in culture. At 28 days, candidate iPSC colonies derived from all three CMSC lines showed well-defined borders and high nuclear-to-cytoplasm ratios making them indistinguishable from undifferentiated H9-ESCs. The reprogramming efficiency of CMSCs into iPSC-like colonies was $0.01 \% \pm 0.005 \%$.

Mature candidate iPSC cell lines derived from reprogrammed CMSCs (herein referred to as CMSC-iPSCs) were successfully transferred onto feeder-free systems, including either Matrigel- or PMEDSAH-coated dishes, for further expansion, subculture, and characterization (Fig. 2B). We confirmed that CMSC-iPSCs expressed high levels of alkaline phosphatase and NANOG (Fig. 2B). Similar to H9ESC, immunofluorescence staining showed uniformly high expression of OCT4, SOX2, SSEA3, SSEA4, TRA-1-60, and TRA-1-81 within CMSC-iPSC colonies (Fig. 2C). In the absence of reprogramming, CMSCs did not display any of these pluripotency markers (data not shown).

Qualitative gene expression analyses of multiple CMSCiPSC lines demonstrated marked upregulation of NANOG as well as increased expression of SOX2 (Fig. 3A). The expression of TERT, KLF4, and cMYC was also increased in CMSC-iPSCs compared to CMSCs (data not shown). Although the expression of NANOG by RT-PCR was uniformly very low among CMSCs, endogenous expression of SOX2 and OCT4 at baseline was often evident (Fig. 3A). Loss of $\alpha$-SMA expression normally present in CMSCs was seen following conversion to iPSCs.

There was an $\sim 1000$-fold upregulation in NANOG in CMSC-iPSCs compared to CMSCs by qPCR (Fig. 3B). There was similar upregulation in OCT4 and SOX2 in CMSCsiPSCs comparable to the levels observed in H9-ESCs. We also observed strong transcription of TERT in CMSC-iPSCs compared to undetectable TERT levels in CMSC controls.

Established CMSC-iPSC lines from all three patients were maintained beyond 15 passages without morphological change. Furthermore, they could be cryopreserved in $10 \%$

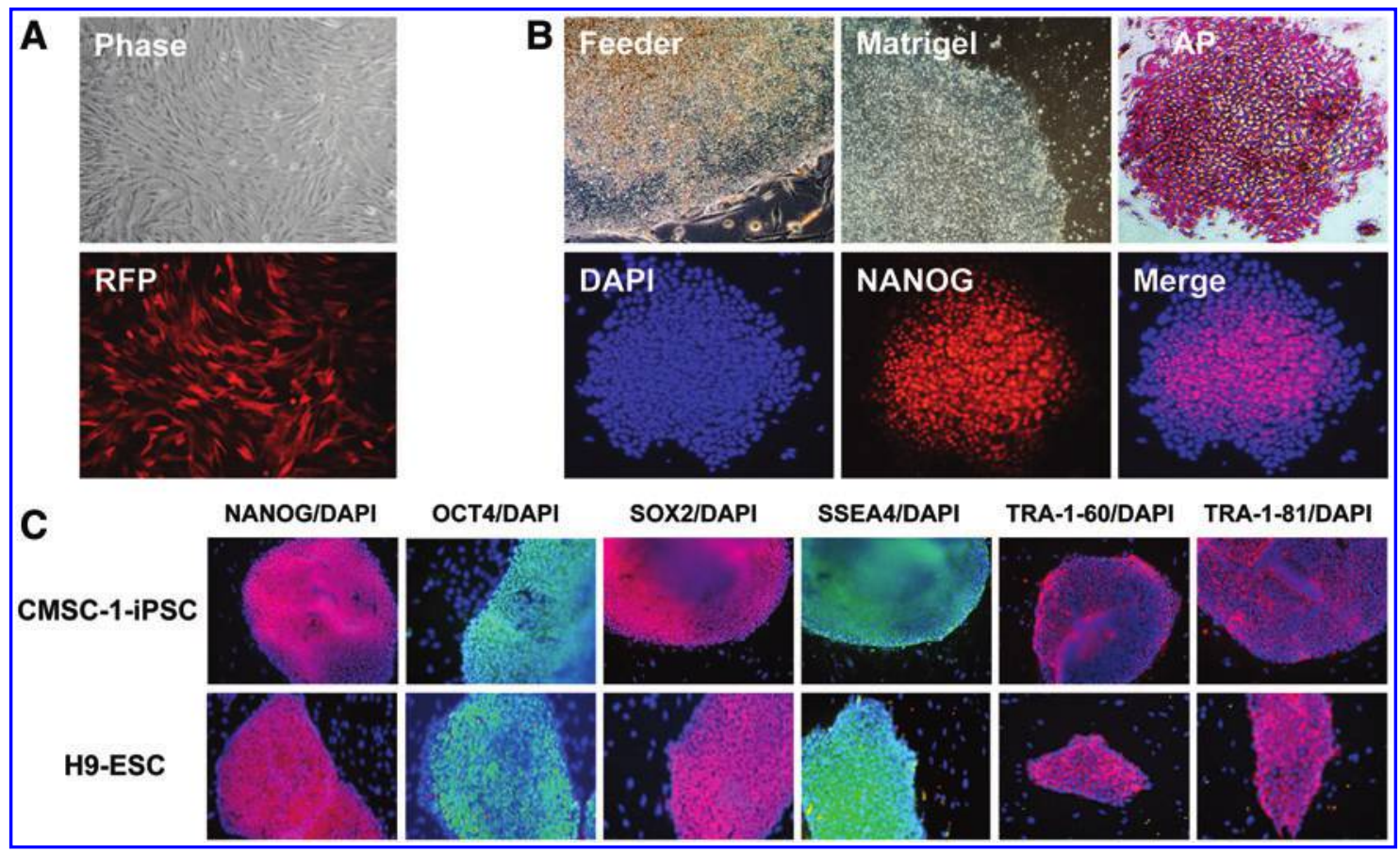

FIG. 2. Characterization of human induced pluripotent stem cells (iPSCs) derived from CMSCs. (A) Phase-contrast photomicrograph of CMSC-1 (upper, $40 \times$ ) following retroviral infection with Yamanaka factors and RFP reporter plasmid on day 2 (lower, magnification, 40×). (B) Representative colony morphology of CMSC-iPSCs grown on mouse embryonic feeders (upper left, Feeder) and Matrigel (upper middle) under brightfield microscopy (magnification, 100×). Early colony with evidence of pluripotency by alkaline phosphatase (AP) staining (upper right, magnification, $20 \times$ ). Superimposition of DAPI and anti-NANOG immunocytochemistry (lower right). (C) Photomicrographs of reprogrammed human CMSC-1 (upper) and H9-ESC controls (lower) on mouse feeder layers, demonstrating expression of the following pluripotency markers: NANOG, OCT4, SOX2, SSEA4, TRA-1-60, and TRA-1-81 (magnification, 20×). DAPI staining in blue. ESC, embryonic stem cell. Color images available online at www.liebertpub.com/tec 


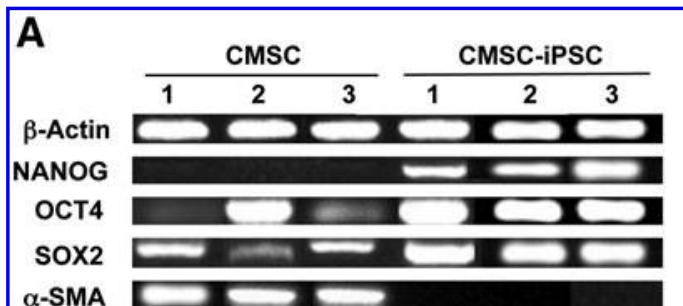

C

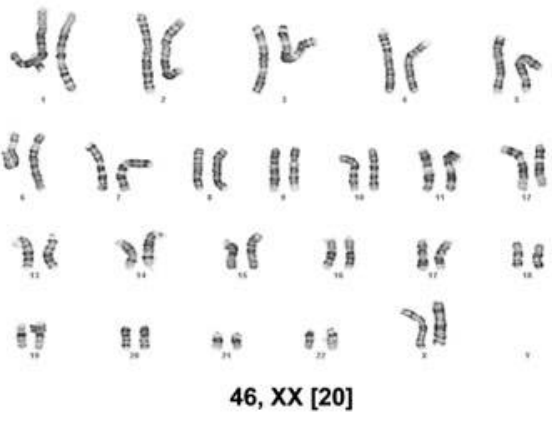

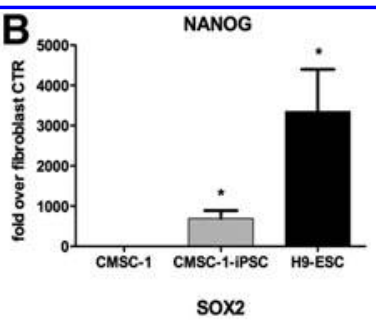
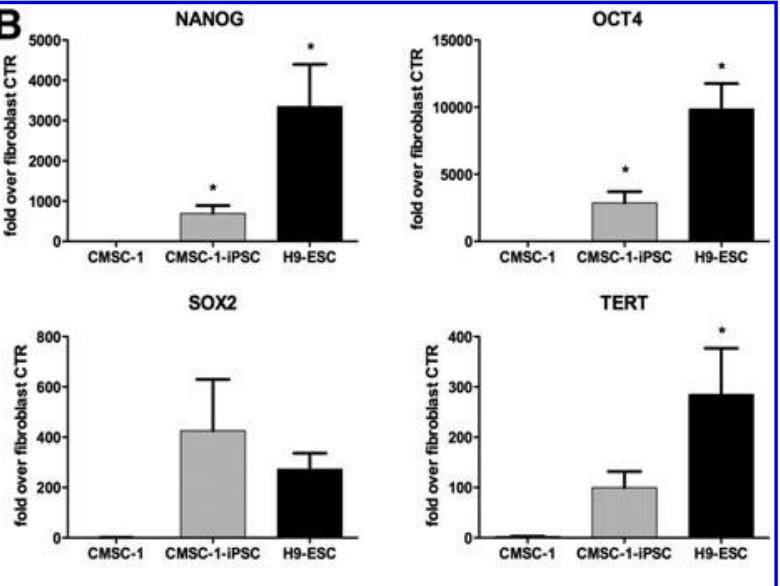

CMYC
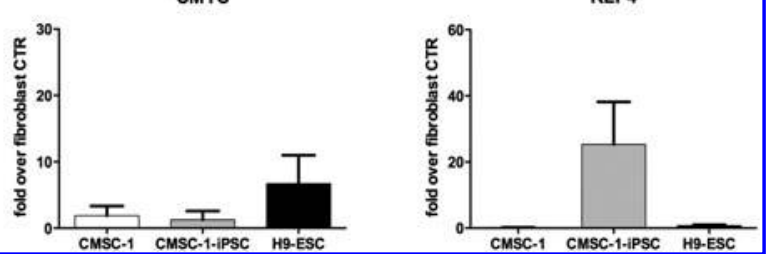

FIG. 3. Genetic analysis of human iPSCs derived from CMSCs. (A) Reverse transcription polymerase chain reaction (RT-PCR) of RNAs from iPSCs showing upregulation of NANOG and downregulation of $\alpha$-SMA. (B) Quantitative analysis by qPCR demonstrating marked upregulation of pluripotency genes, including NANOG, OCT4, SOX2, and TERT, among CMSC-iPSCs $(n=3)$ to levels comparable to that of H9-ESCs. Data were normalized relative to dermal fibroblast controls and presented as the mean $\pm \operatorname{SEM}(n=5$, *denotes $p \leq 0.05)$. (C) Representative karyotype analysis based on 20 G-banded metaphase cells demonstrating normal chromosomes among CMSC-1-iPSCs (passage 5).

DMSO and subsequently thawed with excellent survival and re-expansion capabilities. Cytogenetic analyses revealed a normal karyotype, indicating that these iPSCs remained free of nonclonal aberrations after continuous expansion in culture (Fig. 3C).

In an effort to determine if CMSC-iPSCs were pluripotent in vitro, cells between passages 7 and 9 were allowed to differentiate into EBs. Whereas CMSCs did not form EBs or survive in suspension culture, CMSC-iPSCs were capable of robust $\mathrm{EB}$ formation after 7 days in suspension culture
(Fig. 4A). Between 21 and 28 days, RT-PCR analyses of EBs derived from CMSC-iPSCs showed successful differentiation into endodermal cells by AFP and GATA4 expression, into mesodermal cells by $\alpha$-SMA and DCN expression, and into ectodermal cells by SOX1 and TuJ1 expression (Fig. 4B). With the exception of TuJ1, these genes were expressed at low levels in parental CMSCs. Immunocytochemistry analysis of EBs showed the presence of all three germ layer derivatives (Fig. 4C). Cells at the periphery of the EBs had a flattened morphology that were positive for endoderm
A
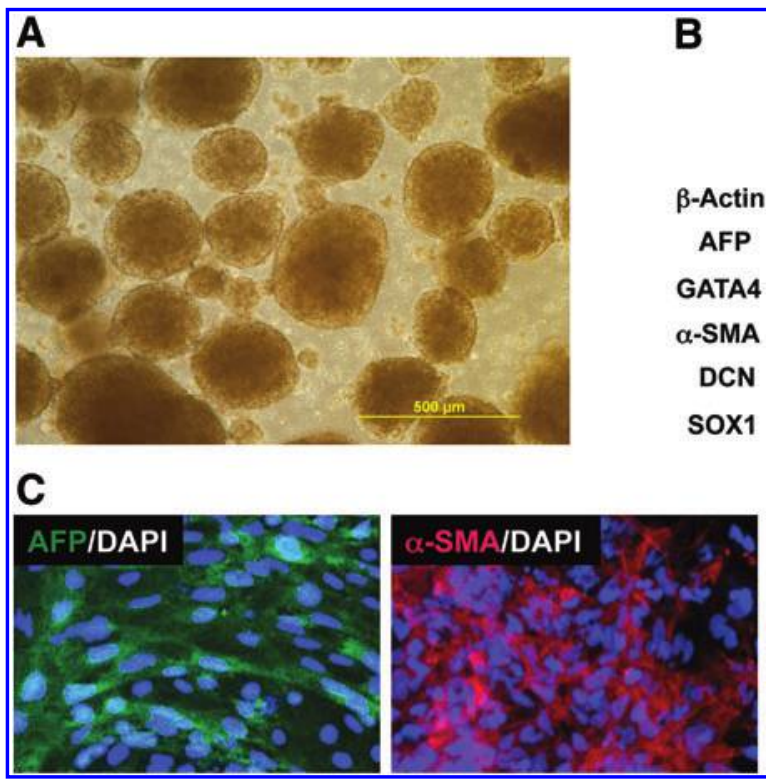

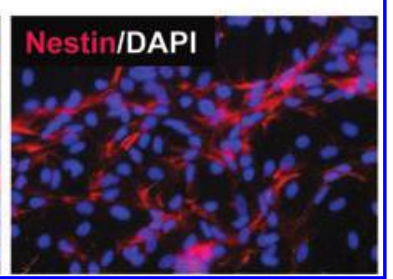

FIG. 4. Representative embryoid body (EB) formation of CMSC-iPSCs. (A) Morphology of EBs (day 14) from CMSC-1-iPSC under brightfield microscopy (scale bar, $500 \mu \mathrm{m}$ ). (B) RTPCR from EB RNA extracted between 21 and 28 days, demonstrating activation of ectodermal, mesodermal, and endodermal genes. (C) Immunofluorescence microscopy of EBs merged with DAPI (in blue) showing expression of AFP (endoderm), $\alpha$-SMA (mesoderm), and Nestin (ectoderm, magnification, $100 \times$ ). Color images available online at www.liebertpub.com/tec 
markers, including AFP, SOX17, and FOXA2. Mesodermal differentiation was confirmed by the presence of $\alpha$-SMA, and ectodermal differentiation was shown by positive Nestin and TuJ1 staining.

Under neurogenic conditions, neurospheres attached and formed neural rosettes typical of neuroepithelial cells within 7 days of adherent culture (Fig. 5A, upper left). These neural precursor cells were subsequently expanded and analyzed at passage 8, showing widespread expression of various NSC markers, including Nestin, PSANCAM, Musashi, and SOX3, by immunofluorescence (Fig. 5A). Undifferentiated CMSC-iPSC controls did not express these neurogenic markers (data not shown). Evidence of early neuronal differentiation was also revealed by TuJ1-positive cells with extended axonal processes (Fig. 5A, lower left). RT-PCR analyses of NSCs differentiated from CMSC-iPSCs (CMSC-iPSC-NSCs) showed the downregulation of NANOG as well as subsequent upregulation of neural progenitor transcription factors, SOX1 (Fig. 5B) and PAX6. Nestin was constitutively expressed at similar levels in both CMSCs and CMSCiPSCs. CMSC-iPSC-NSCs could be cryopreserved and subsequently thawed with excellent survival and re-expansion capabilities.

CMSC-iPSC-NSCs maintained in neurogenic media for up to 2 months were capable of differentiation into more mature neural phenotypes, including neurons and glial cells. Phasecontrast microscopy showed characteristic morphological changes, including extension of neuronal processes (Fig. 5C, upper). Immunofluorescence staining revealed the expression of TuJ1-positive (neuronal) and GFAP-positive (astrocyte) cells without evidence of co-expression (Fig. 5C, lower).

Seven days after induction under cardiogenic conditions, cells began to spontaneously contract (Supplementary Video S1), consistent with mature cardiomyocyte differentiation. Over time, the majority of cells continued to beat in a coordinated rhythmic manner (mean frequency, $129.5 \pm 3.6$ beats per minute at 50 days). Spontaneously contracting could be maintained in culture for over 60 days (Fig. 6A). To confirm directed differentiation into cardiomyocytes, immunofluorescence staining showed strong expression within contracting cells of MYH7, MF20, and $\alpha$-actinin, consistent with cardiac/skeletal myocytes (Fig. 6B). A more differentiated cardiac phenotype was confirmed within CMSC-iPSC-CMs
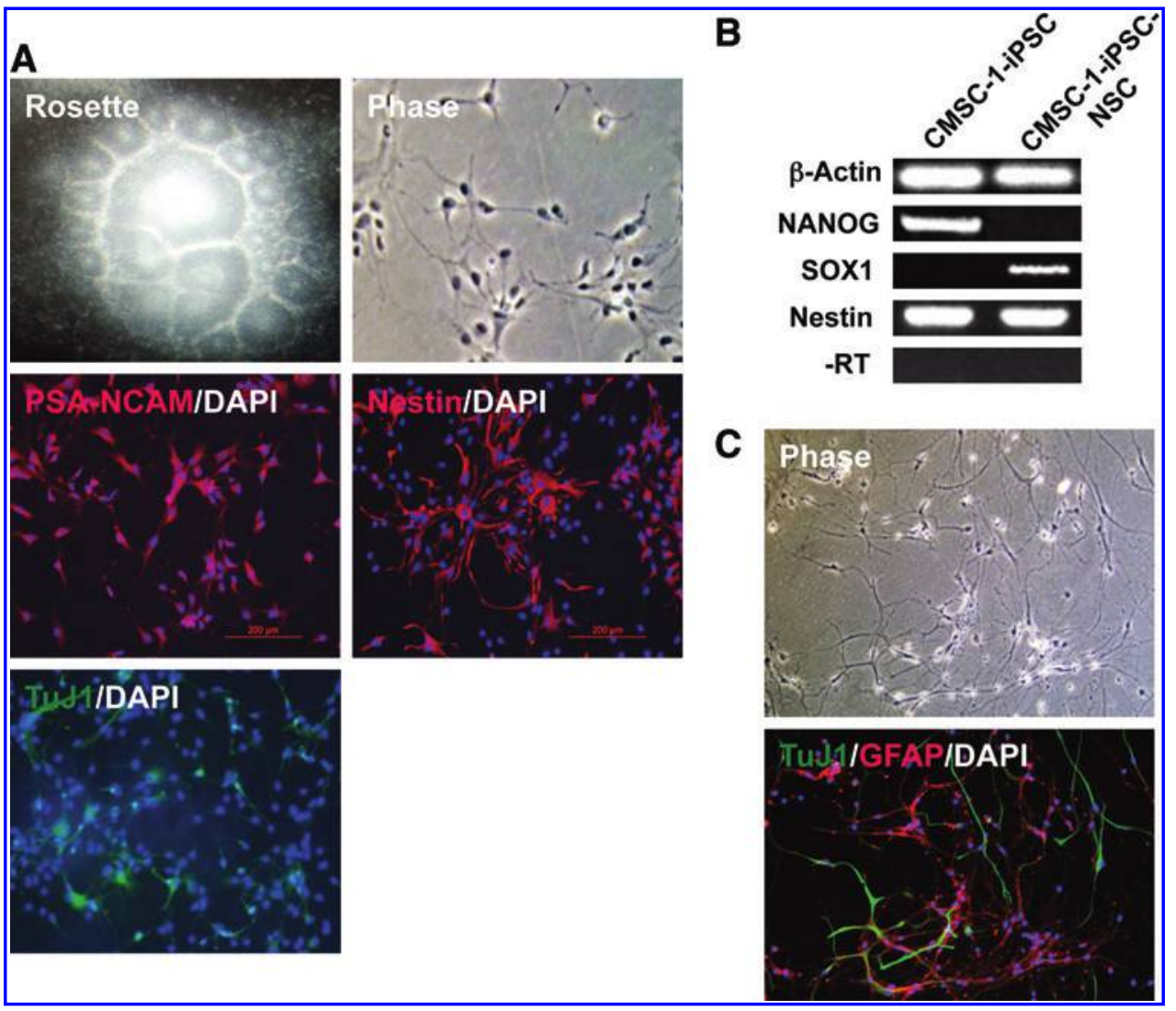

FIG. 5. Directed differentiation of representative CMSC-iPSCs into the neural lineage. (A) Photomicrograph of typical neural rosette formation under brightfield microscopy (upper left, magnification, $4 \times$ ), and morphology of representative neural stem cells (NSCs) under phase microscopy (upper right, magnification, 100×). Merged photomicrographs showing positive immunofluorescence staining for several early neural markers (magnification, 40×), including PSA-NCAM (red, middle left), Nestin (red, middle right), and TuJ1 (green, lower left). DAPI in blue, scale bar, $500 \mu \mathrm{m}$. (B) Upon directed differentiation into the neural lineage, there was loss of NANOG expression but gain in SOX1 expression by RT-PCR. (C) Phase-contrast photomicrograph of more differentiated neural cells (magnification, $20 \times$ ) demonstrating characteristic morphology (upper) that stain positively for TuJ1 (green) or GFAP (red) superimposed with blue DAPI (lower). Color images available online at www.liebertpub.com/tec 


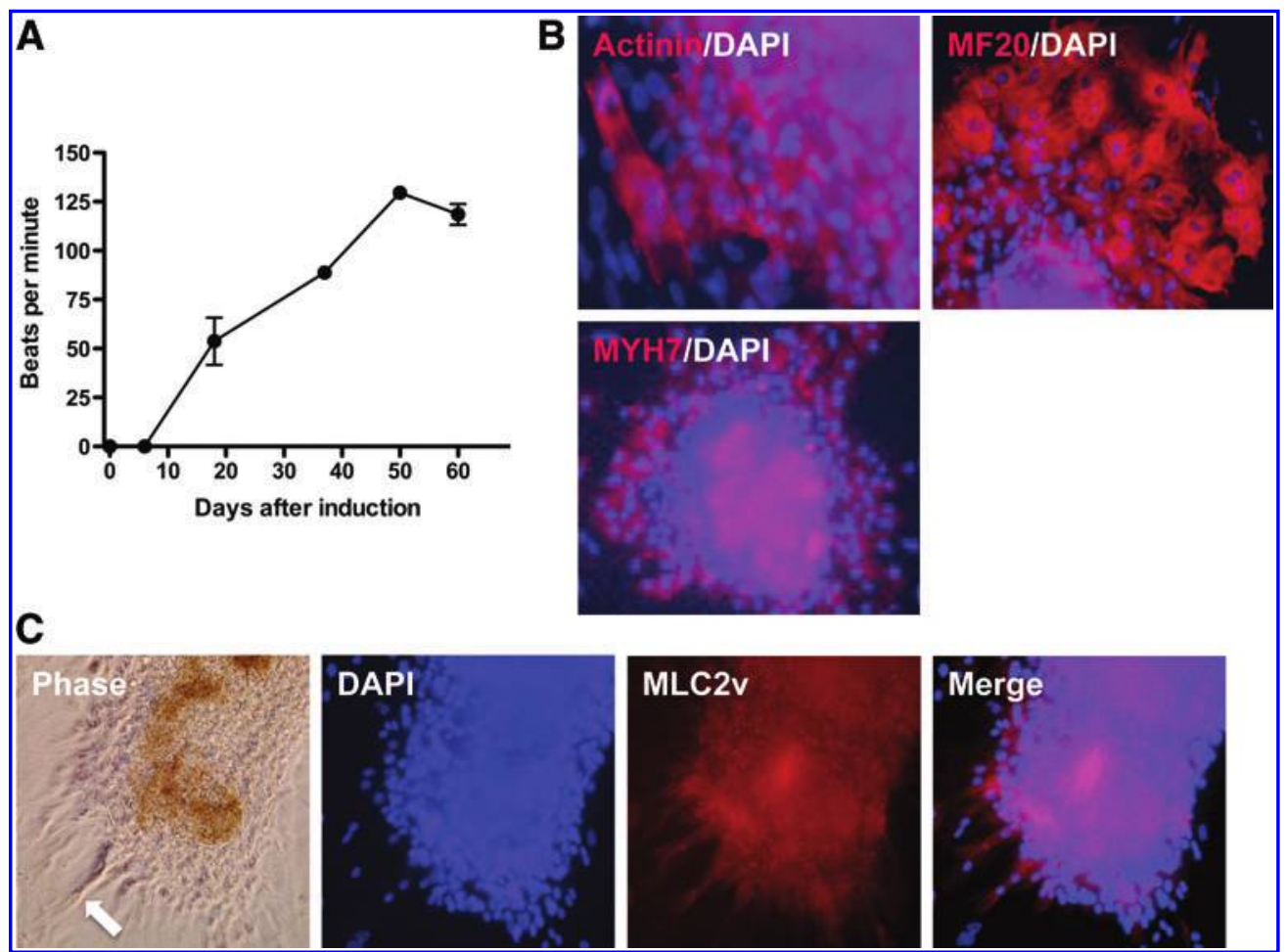

FIG. 6. Directed differentiation of representative CMSC-iPSCs into cardiomyocytes (CMSC-iPSC-CMs). (A) Spontaneous contraction (beats per minute) of CMSC-1-iPSC-CMs maintained under cardiogenic conditions. Data presented as the mean \pm SEM ( $n=4-5$ wells per time point). (B) Positive immunofluorescence staining of cardiomyocytes for myocyte markers (red), including $\alpha$-actinin and MF20 (upper, magnification, $40 \times$ ) as well as MYH7 (lower, magnification, $20 \times$ ), merged with DAPI. (C) Mature ventricular cardiomyocyte differentiation by phase (far left) and immunofluorescence microscopy using an anti-MLC2v antibody (red) superimposed with DAPI (magnification, $20 \times$ ). A muscle fiber is shown by the white arrow. Color images available online at www.liebertpub.com/tec

by robust expression of MLC2v, a protein specific to ventricular cardiomyocytes (Fig. 6C). The absence of NANOG as well as $\alpha$-SMA and RUNX-1, both markers of early mesodermal differentiation, was confirmed by RT-PCR (data not shown). Undifferentiated CMSC-iPSC controls did not express any cardiomyocyte markers (data not shown).

After 3 days under endodermal conditions, robust DE differentiation was evidenced by double-positive immunofluorescence staining of two transcriptional regulators, FOXA1 and SOX17, in a significant proportion of cells (Fig. 7A, lower right). RT-PCR analyses of these DE cells derived from CMSC-iPSCs (CMSC-iPSC-DE) showed the downregulation of NANOG with subsequent upregulation of SOX17 (Fig. 7B). The lack of AFP, a marker seen in primitive endodermal differentiation, was confirmed in CMSCiPSC-DE samples. Undifferentiated CMSC-iPSC controls did not express any endodermal markers (data not shown).

\section{Discussion}

In this study, we successfully derived new human iPSC lines from term fetal chorionic mesenchyme and demonstrated

\section{A}
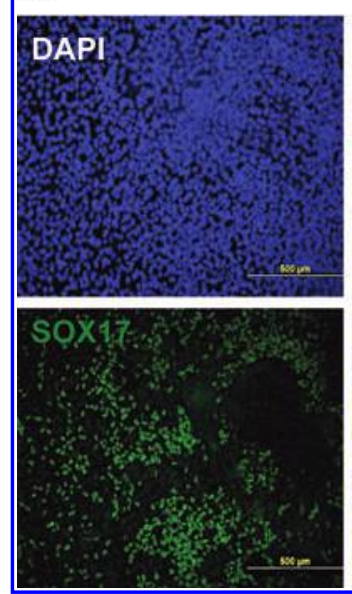

B
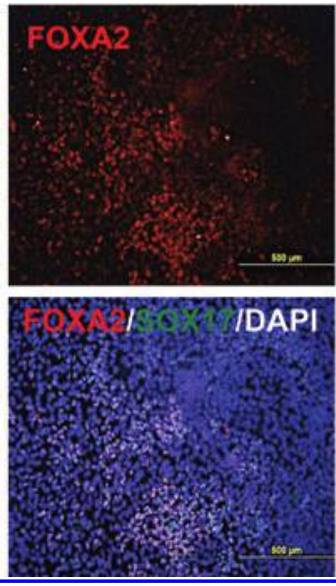

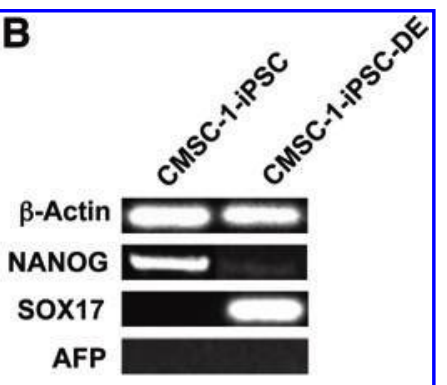

FIG. 7. Directed differentiation of representative CMSC-iPSCs into definitive endoderm (DE). (A) Immunofluorescence analysis of CMSC-1iPSC-DE demonstrating double-positive staining of two definitive endoderm transcriptional regulators, FOXA2 (red) and SOX17 (green), superimposed with DAPI (lower right, magnification, $20 \times)$. Scale bar, $500 \mu \mathrm{m}$. (B) RT-PCR showing upregulation in SOX17 with concomitant downregulation in NANOG following endodermal induction. The DE phenotype is further supported by the absence of AFP. Color images available online at www.liebertpub.com/tec 
continuous expansion under feeder-free culture conditions, including PMEDSAH, a defined synthetic polymer matrix. The pluripotency of our CMSC-iPSCs, as revealed by controlled in vitro differentiation of CMSCs into each of the three germ layers, provides proof of concept of their potential in maternalfetal research and clinical applications. To our knowledge, only one other study has successfully described iPSC reprogramming of term human chorionic cells, but these cells were incompletely characterized in vitro by pluripotency markers and functional assays. $^{20}$

Despite their heterogeneity and increased expression of selected pluripotent markers, CMSCs are classified within the family of placental MSCs based on criteria established by the first international Workshop on Placenta Derived Stem Cells. ${ }^{21}$ In most experimental models, MSCs and their related cell types, including CD117-positive amniotic fluid stem cells, have enhanced plasticity but appear to exert the bulk of their actions in vivo as paracrine mediators via the secretion of various factors that support the growth, survival, or characteristics of other cells, rather than by direct terminal differentiation into new tissue. ${ }^{22}$ Unlike true pluripotent stem cells, extended in vitro culture of placental-based MSCs has generally been associated with cell senescence and reduced plasticity beyond the mesodermal lineages. ${ }^{23}$

Although the reprogramming efficiency observed in our study was relatively low, methods to enhance the iPSC derivation of CMSCs and other fetal cell types remain ongoing. Regardless, the overall findings raise awareness regarding the potential benefits of banking placenta chorionic cells for the future patient-specific therapeutic applications. In most cases, human chorionic cells can be easily established and expanded in primary culture from a small specimen. In addition to providing a cellular substrate for iPSC reprogramming, our work confirms that CMSCs are of fetal origin and therefore would be available for gene and other cell-based therapies later in life analogous to what is already being done with umbilical cord blood banking for hematopoietic stem cell transplantation.

Although the use of fetal-derived somatic tissues as an alternative cell source for iPSC reprogramming is not entirely new, ${ }^{7,8,24-27}$ we believe that CMSCs are particularly novel, unique, and practical cells for many perinatal tissue engineering applications that would utilize pluripotent stem cell technologies. Compared to many other possible cells that could be obtained in this patient population, placenta-derived MSCs can be easily expanded in vitro to generate a large number of somatic cells, can be reliably reprogrammed with only four transcription factors, have epigenetic memory closer to a pluripotent state, and have enhanced immunomodulatory properties even after iPSC induction that may be advantageous for allogeneic applications. ${ }^{28}$ Experience in our laboratory, among others, has shown that MSCs are less reliably isolated from umbilical cord blood samples. ${ }^{29,30}$ Moreover, cord blood hematopoietic cells are notoriously difficult to expand in vitro, a property that has already limited their therapeutic potential in clinical hematopoietic stem cell transplantation. Four-factor iPSC reprogramming efficiency rates as low as $0.0008 \%$ have been reported from mononuclear cells isolated from blood samples. ${ }^{31}$ Finally, blood-derived somatic cells may also have increased epigenetic memory biasing them toward hematopoietic differentiation after cellular reprogramming. ${ }^{32}$
Ultimately, we anticipate that the many unique aspects of human CMSCs, particularly their endogenous expression of OCT4, Nestin, and other markers, suggest that these cells may play a key role in unlocking some of the fundamental questions with regard to epigenetic memory, conversion to alternate cell phenotypes, and the development of transgenefree cell-based therapeutic applications in infants and young children with debilitating diseases. Differentiated cell types derived from CMSC-iPSCs have enormous potential as valuable in vitro platforms for disease modeling and testing of pharmacological agents in children with congenital anomalies. Pediatric disorders, including congenital heart disease, pulmonary hypoplasia, and neurological development disorders, are typically diagnosed prenatally and therefore could be further investigated in these models once the child is born. In addition, purified differentiated populations of cells derived from CMSC-iPSCs in vitro may facilitate the development of autologous tissue engineering and other cell-based therapies for some of the most difficult perinatal disorders treated in neonatal intensive care units worldwide. ${ }^{33}$ These diseases, all of which are manifested by damage or loss of organ function, include neonatal hypoxicischemic encephalopathy, cerebral palsy, spina bifida, hypoplastic left heart syndrome, and fulminant hepatic failure. $^{34}$

Whereas the delivery of autologous CMSC-iPSCs might be appealing for certain regenerative medicine applications, allogeneic transplantation using cryopreserved cells derived from human leukocyte antigen (HLA)-matched CMSCiPSCs stored within a biorepository represents another viable alternative. An allogeneic approach might offer some additional cost advantages, enabling the initiation of cellbased treatments earlier in infancy. The ability to use HLA-matched cells "off-the-shelf" without the need for immunosuppression might also be feasible in this setting given the known immunoregulatory properties of fetalderived mesenchymal cells. ${ }^{28,35}$

\section{Conclusions}

This study demonstrates the feasibility of term placental chorion as a noninvasive alternative cell source for perinatal iPSC derivation. Because human iPSCs derived from placental chorion have virtually identical properties to that of human ESCs, they have enormous potential in fetal tissue engineering and regenerative medicine research applications and may provide additional insights regarding the reprogramming capabilities of extra-embryonic tissues as they relate to developmental ontogeny.

\section{Acknowledgments}

This study was supported by the Robert Wood Johnson Foundation (Harold Amos Scholarship to Dr. Kunisaki) and the American College of Surgeons (James Carrico Award to Dr. Kunisaki). The authors thank Jeannie Kreutzman, RN, MSN, CPNP, for facilitating the collection of placenta samples, and Paul H. Krebsbach, DDS, PhD, for providing assistance with feeder-free polymer matrices.

\section{Disclosure Statement}

No competing financial interests exist. 


\section{References}

1. Takahashi, K., and Yamanaka, S. Induction of pluripotent stem cells from mouse embryonic and adult fibroblast cultures by defined factors. Cell 126, 663, 2006.

2. Yu, J., Vodyanik, M.A., Smuga-Otto, K., AntosiewiczBourget, J., Frane, J.L., Tian, S., Nie, J., Jonsdottir, G.A., Ruotti, V., Stewart, R., Slukvin, II, and Thomson, J.A. Induced pluripotent stem cell lines derived from human somatic cells. Science 318, 1917, 2007.

3. Park, I.H., Arora, N., Huo, H., Maherali, N., Ahfeldt, T., Shimamura, A., Lensch, M.W., Cowan, C., Hochedlinger, K., and Daley, G.Q. Disease-specific induced pluripotent stem cells. Cell 134, 877, 2008.

4. Sun, N., Panetta, N.J., Gupta, D.M., Wilson, K.D., Lee, A., Jia, F., Hu, S., Cherry, A.M., Robbins, R.C., Longaker, M.T., and $\mathrm{Wu}$, J.C. Feeder-free derivation of induced pluripotent stem cells from adult human adipose stem cells. Proc Natl Acad Sci U S A 106, 15720, 2009.

5. Giorgetti, A., Montserrat, N., Aasen, T., Gonzalez, F., Rodriguez-Piza, I., Vassena, R., Raya, A., Boue, S., Barrero, M.J., Corbella, B.A., Torrabadella, M., Veiga, A., and Izpisua Belmonte, J.C. Generation of induced pluripotent stem cells from human cord blood using OCT4 and SOX2. Cell Stem Cell 5, 353, 2009.

6. Jones, G.N., Moschidou, D., Puga-Iglesias, T.I., Kuleszewicz, K., Vanleene, M., Shefelbine, S.J., Bou-Gharios, G., Fisk, N.M., David, A.L., De Coppi, P., and Guillot, P.V. Ontological differences in first compared to third trimester human fetal placental chorionic stem cells. PLoS One 7, e43395, 2012.

7. Cai, J., Li, W., Su, H., Qin, D., Yang, J., Zhu, F., Xu, J., He, W., Guo, X., Labuda, K., Peterbauer, A., Wolbank, S., Zhong, M., Li, Z., Wu, W., So, K.F., Redl, H., Zeng, L., Esteban, M.A., and Pei, D. Generation of human induced pluripotent stem cells from umbilical cord matrix and amniotic membrane mesenchymal cells. J Biol Chem 285, 11227, 2010.

8. Fujioka, T., Shimizu, N., Yoshino, K., Miyoshi, H., and Nakamura, Y. Establishment of induced pluripotent stem cells from human neonatal tissues. Hum Cell 23, 113, 2010.

9. Nazarov, I., Lee, J.W., Soupene, E., Etemad, S., Knapik, D., Green, W., Bashkirova, E., Fang, X., Matthay, M.A., Kuypers, F.A., and Serikov, V.B. Multipotent stromal stem cells from human placenta demonstrate high therapeutic potential. Stem Cells Transl Med 1, 359, 2012.

10. Dominici, M., Le Blanc, K., Mueller, I., Slaper-Cortenbach, I., Marini, F., Krause, D., Deans, R., Keating, A., Prockop, D., and Horwitz, E. Minimal criteria for defining multipotent mesenchymal stromal cells. The International Society for Cellular Therapy position statement. Cytotherapy 8, 315, 2006.

11. Villa-Diaz, L.G., Brown, S.E., Liu, Y., Ross, A.M., Lahann, J., Parent, J.M., and Krebsbach, P.H. Derivation of mesenchymal stem cells from human induced pluripotent stem cells cultured on synthetic substrates. Stem Cells 30, 1174, 2012.

12. Bentley, J.K., Popova, A.P., Bozyk, P.D., Linn, M.J., Baek, A.E., Lei, J., Goldsmith, A.M., and Hershenson, M.B. Ovalbumin sensitization and challenge increases the number of lung cells possessing a mesenchymal stromal cell phenotype. Respir Res 11, 127, 2010.

13. Park, I.H., and Daley, G.Q. Human iPS cell derivation/ reprogramming. Curr Protoc Stem Cell Biol Chapter 4, Unit 4A.1, 2009.
14. Itskovitz-Eldor, J., Schuldiner, M., Karsenti, D., Eden, A., Yanuka, O., Amit, M., Soreq, H., and Benvenisty, N. Differentiation of human embryonic stem cells into embryoid bodies compromising the three embryonic germ layers. Mol Med 6, 88, 2000.

15. Livak, K.J., and Schmittgen, T.D. Analysis of relative gene expression data using real-time quantitative PCR and the 2(-Delta Delta C(T)) method. Methods 25, 402, 2001.

16. Mak, S.K., Huang, Y.A., Iranmanesh, S., Vangipuram, M., Sundararajan, R., Nguyen, L., Langston, J.W., and Schule, B. Small molecules greatly improve conversion of humaninduced pluripotent stem cells to the neuronal lineage. Stem Cells Int 2012, 140427, 2012.

17. Zhang, J., Klos, M., Wilson, G.F., Herman, A.M., Lian, X., Raval, K.K., Barron, M.R., Hou, L., Soerens, A.G., Yu, J., Palecek, S.P., Lyons, G.E., Thomson, J.A., Herron, T.J., Jalife, J., and Kamp, T.J. Extracellular matrix promotes highly efficient cardiac differentiation of human pluripotent stem cells: the matrix sandwich method. Circ Res 111, 1125, 2012.

18. D’Amour, K.A., Agulnick, A.D., Eliazer, S., Kelly, O.G., Kroon, E., and Baetge, E.E. Efficient differentiation of human embryonic stem cells to definitive endoderm. Nat Biotechnol 23, 1534, 2005.

19. Spence, J.R., Mayhew, C.N., Rankin, S.A., Kuhar, M.F., Vallance, J.E., Tolle, K., Hoskins, E.E., Kalinichenko, V.V., Wells, S.I., Zorn, A.M., Shroyer, N.F., and Wells, J.M. Directed differentiation of human pluripotent stem cells into intestinal tissue in vitro. Nature 470, 105, 2011.

20. Esteban, M.A., Wang, T., Qin, B., Yang, J., Qin, D., Cai, J., Li, W., Weng, Z., Chen, J., Ni, S., Chen, K., Li, Y., Liu, X., Xu, J., Zhang, S., Li, F., He, W., Labuda, K., Song, Y., Peterbauer, A., Wolbank, S., Redl, H., Zhong, M., Cai, D., Zeng, L., and Pei, D. Vitamin C enhances the generation of mouse and human induced pluripotent stem cells. Cell Stem Cell 6, 71, 2010.

21. Parolini, O., Alviano, F., Bagnara, G.P., Bilic, G., Buhring, H.J., Evangelista, M., Hennerbichler, S., Liu, B., Magatti, M., Mao, N., Miki, T., Marongiu, F., Nakajima, H., Nikaido, T., Portmann-Lanz, C.B., Sankar, V., Soncini, M., Stadler, G., Surbek, D., Takahashi, T.A., Redl, H., Sakuragawa, N., Wolbank, S., Zeisberger, S., Zisch, A., and Strom, S.C. Concise review: isolation and characterization of cells from human term placenta: outcome of the first international Workshop on Placenta Derived Stem Cells. Stem Cells 26, 300, 2008.

22. Prasongchean, W., Bagni, M., Calzarossa, C., De Coppi, P., and Ferretti, P. Amniotic fluid stem cells increase embryo survival following injury. Stem Cells Dev 21, 675, 2012.

23. Macias, M.I., Grande, J., Moreno, A., Dominguez, I., Bornstein, R., and Flores, A.I. Isolation and characterization of true mesenchymal stem cells derived from human term decidua capable of multilineage differentiation into all 3 embryonic layers. Am J Obstet Gynecol 203, 495 e9, 2010.

24. Ye, L., Chang, J.C., Lin, C., Sun, X., Yu, J., and Kan, Y.W. Induced pluripotent stem cells offer new approach to therapy in thalassemia and sickle cell anemia and option in prenatal diagnosis in genetic diseases. Proc Natl Acad Sci U S A 106, 9826, 2009.

25. Li, C., Zhou, J., Shi, G., Ma, Y., Yang, Y., Gu, J., Yu, H., Jin, S., Wei, Z., Chen, F., and Jin, Y. Pluripotency can be rapidly and efficiently induced in human amniotic fluidderived cells. Hum Mol Genet 18, 4340, 2009. 
26. Galende, E., Karakikes, I., Edelmann, L., Desnick, R.J., Kerenyi, T., Khoueiry, G., Lafferty, J., McGinn, J.T., Brodman, M., Fuster, V., Hajjar, R.J., and Polgar, K. Amniotic fluid cells are more efficiently reprogrammed to pluripotency than adult cells. Cell Reprogram 12, 117, 2010.

27. Zhao, H.X., Li, Y., Jin, H.F., Xie, L., Liu, C., Jiang, F., Luo, Y.N., Yin, G.W., Wang, J., Li, L.S., Yao, Y.Q., and Wang, X.H. Rapid and efficient reprogramming of human amnion-derived cells into pluripotency by three factors OCT4/SOX2/NANOG. Differentiation 80, 123, 2010.

28. Liu, P., Chen, S., Li, X., Qin, L., Huang, K., Wang, L., Huang, W., Li, S., Jia, B., Zhong, M., Pan, G., Cai, J., and Pei, D. Low immunogenicity of neural progenitor cells differentiated from induced pluripotent stem cells derived from less immunogenic somatic cells. PLoS One 8, e69617, 2013.

29. Bieback, K., and Brinkmann, I. Mesenchymal stromal cells from human perinatal tissues: from biology to cell therapy. World J Stem Cells 2, 81, 2010.

30. Kern, S., Eichler, H., Stoeve, J., Kluter, H., and Bieback, K. Comparative analysis of mesenchymal stem cells from bone marrow, umbilical cord blood, or adipose tissue. Stem Cells 24, 1294, 2006.

31. Loh, Y.H., Hartung, O., Li, H., Guo, C., Sahalie, J.M., Manos, P.D., Urbach, A., Heffner, G.C., Grskovic, M., Vigneault, F., Lensch, M.W., Park, I.H., Agarwal, S., Church, G.M., Collins, J.J., Irion, S., and Daley, G.Q. Reprogramming of $\mathrm{T}$ cells from human peripheral blood. Cell Stem Cell 7, 15, 2010.

32. Kim, K., Zhao, R., Doi, A., Ng, K., Unternaehrer, J., Cahan, P., Huo, H., Loh, Y.H., Aryee, M.J., Lensch, M.W., Li, H., Collins, J.J., Feinberg, A.P., and Daley, G.Q. Donor cell type can influence the epigenome and differentiation potential of human induced pluripotent stem cells. Nat Biotechnol 29, 1117, 2011.

33. Fauza, D. Amniotic fluid and placental stem cells. Best Pract Res Clin Obstet Gynaecol 18, 877, 2004.

34. Titomanlio, L., Kavelaars, A., Dalous, J., Mani, S., El Ghouzzi, V., Heijnen, C., Baud, O., and Gressens, P. Stem cell therapy for neonatal brain injury: perspectives and challenges. Ann Neurol 70, 698, 2011.

35. Bailo, M., Soncini, M., Vertua, E., Signoroni, P.B., Sanzone, S., Lombardi, G., Arienti, D., Calamani, F., Zatti, D., Paul, P., Albertini, A., Zorzi, F., Cavagnini, A., Candotti, F., Wengler, G.S., and Parolini, O. Engraftment potential of human amnion and chorion cells derived from term placenta. Transplantation 78, 1439, 2004.

Address correspondence to: Shaun M. Kunisaki, MD, MSc Department of Surgery C.S. Mott Children's Hospital Von Voigtlander Women's Hospital University of Michigan Medical School 1540 E. Hospital Drive, SPC 4211 Ann Arbor, MI 48109

E-mail: shaunkun@umich.edu

Received: August 5, 2013 Accepted: December 12, 2013 Online Publication Date: February 28, 2014 


\section{This article has been cited by:}

1. Melissa Vanover, Aijun Wang, Diana Farmer. 2017. Potential clinical applications of placental stem cells for use in fetal therapy of birth defects. Placenta 59, 107-112. [Crossref]

2. Antonietta R. Silini, Sara Cancelli, Patrizia Bonassi Signoroni, Anna Cargnoni, Marta Magatti, Ornella Parolini. 2017. The dichotomy of placenta-derived cells in cancer growth. Placenta 59, 154-162. [Crossref]

3. Yuping Wang. 2017. Vascular Biology of the Placenta, Second Edition. Colloquium Series on Integrated Systems Physiology: From Molecule to Function 9:3, i. [Crossref]

4. George T.-J. Huang, Ikbale El Ayachi, Xiao-Ying Zou. Induced pluripotent stem cell technologies for tissue engineering 1-19. [Crossref]

5. Vikram Sabapathy, Sanjay Kumar. 2016. hiPSC-derived iMSCs: NextGen MSCs as an advanced therapeutically active cell resource for regenerative medicine. Journal of Cellular and Molecular Medicine 20:8, 1571-1588. [Crossref]

6. Faezeh Faghihi, Esmaeil Mirzaei, Jafar Ai, Abolfazl Lotfi, Forough Azam Sayahpour, Somayeh Ebrahimi Barough, Mohammad Taghi Joghataei. 2016. Differentiation Potential of Human Chorion-Derived Mesenchymal Stem Cells into Motor Neuron-Like Cells in Two- and Three-Dimensional Culture Systems. Molecular Neurobiology 53:3, 1862-1872. [Crossref]

7. Williams Corin, Guldberg Robert E.. 2016. Tissue Engineering for Pediatric Applications. Tissue Engineering Part A 22:3-4, 195-196. [Abstract] [Full Text HTML] [Full Text PDF] [Full Text PDF with Links]

8. Gabriela Kmiecik, Valentina Spoldi, Antonietta Silini, Ornella Parolini. 2015. Current View on Osteogenic Differentiation Potential of Mesenchymal Stromal Cells Derived from Placental Tissues. Stem Cell Reviews and Reports 11:4, 570-585. [Crossref] 